\title{
Comprensión de textos y estilos de aprendizaje en los estudiantes universitarios de huancayo
}

\section{Yaćhana lulaynin killkakuna yaćhanawan hatunyaćhaywasip yaćhapakuninkuna huancayopi}

Recepción: 08 julio 2020 Corregido: 16 agosto 2020 Aprobación: 01 noviembre 2020

Irina Adriana Palacios Canorio

Nacionalidad: Peruana / Instituto Nacional de Enfermedades Neoplasicas, Departamento de Radioterapia. Correo: irina.palacios.c@upch.pe / ORCID: https://orcid.org/oooo-0o03-3113-1647

Patricia Ninfa Zegarra Casas Nacionalidad: Peruana / Universidad Continental. Correo: pzegarracasas@gmail.com / ORCID: https://orcid.org/oooo-0002-5116-1141

Jhonny Paúl Castro Mucha Nacionalidad: Peruana / Universidad Continental. Correo:jcastromu@continental.edu.pe / ORCID: https://orcid.org/oooo-0002-4856-7650

Marco Antonio Palacios Villanes

Nacionalidad: Peruano / Universidad Nacional del Centro del Perú. Correo: marcoantoniopalaciosvillanes@gmail.com / ORCID: http://orcid.org/0000-0002-2669-0092

\section{Resumen}

La interrogante a responder fue: ¿Qué diferencias significativas de comprensión de textos presentan los estudiantes universitarios de Huancayo, según el estilo de aprendizaje, edad, género, condición económica y condición familiar? El objetivo central ha sido: Establecer las diferencias significativas de comprensión de textos que presentan los estudiantes universitarios de Huancayo, según el estilo de aprendizaje, edad, género, condición económica y condición familiar. Se tuvo como hipótesis general: Los estudiantes universitarios de Huancayo presentan diferencias significativas de comprensión de textos, según el estilo de aprendizaje, edad, género, condición económica y condición familiar. Investigación cuantitativa, transversal, con diseño descriptivo comparativo. Muestra no probabilística e intencionada, constituida por 300 estudiantes, agrupados por género, edad, condición económica y condición familiar. La comprensión de textos se midió con la prueba pedagógica LISIN de Palacios (2005) y los estilos de aprendizaje con el cuestionario de Honey - Alonso (CHAE). Los resultados evidencian que los estudiantes universitarios de Huancayo presentan un nivel bajo de comprensión de textos y los estilos de aprendizaje no evidencian una relación directa con la comprensión de textos.

\section{Palabras clave:}

Comprensión, textos y estilos de aprendizaje

\section{Lisichiku limaykuna:}

Allikyaćhana, yaćhayplulayninkuna killkakunawan. 


\title{
Understanding of Texts and Styles of Learning in the University Students de Huancayo
}

\begin{abstract}
The question to answer was: What you differ significant of understanding of texts the university students of Huancayo present, according to the learning style, age, gender, economic condition and family condition? The central objective has been: The significant differences of understanding of texts that the university students of Huancayo present to settle down, according to the learning style, age, gender, economic condition and family condition. One had as general hypothesis: The university students of Huancayo present significant differences of understanding of texts, according to the learning style, age, gender, economic condition and family condition. Quantitative, traverse investigation, with comparative descriptive design. It shows non probabilistic and deliberate, constituted by 300 students, contained by gender, age, economic condition and family condition. The understanding of texts was measured with the pedagogic test LISIN of Palacios (2005) and the learning styles with the questionnaire of Honey - Alonso (CHAE). The results evidence that the university students of Huancayo present a low level of understanding of texts and the learning styles don't evidence a direct relationship with the understanding of texts.
\end{abstract}

\section{Compreensão de textos e estilos de aprendizagem nos estudantes universitários de Huancayo}

\section{Resumo}

A questão a ser respondida foi: Que diferenças significativas na compreensão de textos apresentam os estudantes universitários de Huancayo, de acordo com o estilo de aprendizagem, idade, gênero, condição econômica e condição familiar? O objetivo principal foi: estabelecer as diferenças significativas na compreensão dos textos apresentados pelos universitários de Huancayo, de acordo com o estilo de aprendizagem, idade, gênero, condição econômica e condição familiar. Hipótese geral: os estudantes universitários de Huancayo apresentam diferenças significativas na compreensão dos textos, de acordo com o estilo de aprendizagem, idade, gênero, condição econômica e condição familiar. Pesquisa quantitativa, transversal, com desenho descritivo comparativo. Amostra não probabilística e intencional, composta por 300 estudantes, agrupados por gênero, idade, condição econômica e condição familiar. A compreensão do texto foi medida com o teste pedagógico LISIN de Palacios (2005) e os estilos de aprendizagem com o questionário Honey - Alonso (CHAE). Os resultados mostram que os universitários de Huancayo apresentam um baixo nível de compreensão de textos e os estilos de aprendizagem não evidenciam relação direta com a compreensão de textos.

\section{Datos de los autores}

Irina Adriana Palacios Canorio es investigadora en radioterapia en el Instituto Nacional de Enfermedades Neoplasicas. Lic en Radioterapia, Universidad Peruana Cayetano Heredia.

Patricia Ninfa Zegarra Casas es Docente del área de Comunicación. Mg. en Educación. Con mención en Docencia y Gestión Educativa por la Universidad Privada Cesar Vallejo, Perú.

Jhonny Paúl Castro Mucha es investigador y Docente de Comunicación Efectiva y Habilidades Comunicativas. Maestro en Problemas de Aprendizaje por la Universidad Nacional Enrique Guzmán y Valle "La Cantuta".

Marco Antonio Palacios Villanes es investigador y docente de Español y Literatura. Dr. Ciencias de la Educación por la Universidad de Educación Enrique Guzmán y Valle "La Cantuta”, Perú. Magister en Didáctica Universitaria por la Universidad Nacional del Centro del Perú. 


\section{Introducción}

La comprensión lectora es un problema, que no solo atañe a la educación peruana sino a todo el mundo. Los déficits en la lectura y sobre todo en la comprensión se han ido incrementando, es posible que este fenómeno se deba a la influencia de muchos factores, entre ellos las redes sociales y la internet. En la actualidad, los esfuerzos por obtener la información son mínimos, los nativos digitales solo se preocupan por acceder a la información que se encuentran en la web sin verificar la valía de estos y le dan credibilidad a cualquier dicho que circula en la internet.

Por otro lado, el hecho de tener la información a la mano, sin que haya pasado por el filtro de veracidad; es más, escueta y carente de argumentos se ha convertido en una moda que se practica en las redes sociales. Los jóvenes del millenium y los nativos digitales se están habituando a leer pequeños trozos de información y lo dan, por cierto. No se busca la fuente, ni menos se practica la investigación o simplemente buscan una lectura complementaria que sacie su curiosidad. Nos estamos contentando a no leer y menos a indagar la información pertinente.

En el plano universitario esto se ha convertido en una práctica desmedida. Los universitarios se contentan con las primeras informaciones de las primeras ventanas expuestas en la web. Información carente de fundamentos, sin autoría y sin fundamentos teóricos sólidos; pero como no quieren esforzarse se limitan copiando y difundiendo información irrelevante (Palacios, 2018).

Bueno, en estos últimos años, mucho se ha hablado acerca de la problemática de la comprensión de lectura. También se sabe que existe muchas variables que influyen en su resultado, algunos mínimamente y otros de manera preponderante. En suma, la comprensión lectora es la consecuencia de lo que se hizo y no se hizo tanto en el seno familiar como en el plano escolar. Como se sabe no es un tema nuevo, sin embargo, la preocupación por estudiarlo se ha incrementado en esta última década, y es posible que se siga insistiendo en los próximos años, debido a que los resultados, en todos los niveles, es deficiente y que solo existe un dominio literal, memorístico repetitivo y sin duda la respuesta tácita es como mejorarlo (Zavala, 2008).

La teoría interactiva direcciona la comprensión de lectura en una interrelación entre el lector y el texto dentro de un contexto sociocultural; por cierto, involucra los saberes previos, vocabulario, motivaciones, intereses, etc., del lector y la complejidad textual del propio texto, es ahí donde se produce el fenómeno comprensivo. Como consecuencia, la comprensión no solo involucra la reproducción literal del texto en forma global; sino que establece las conexiones de los elementos lingüísticos, las formas como se han combinado para establecer construcciones superiores, asimismo descubrir la macroestructura textual, desentrañando el significado profundo, y arribando a inferencias pertinentes que posibiliten nuevas relaciones textuales y creaciones.

Si bien es cierto, el texto, por sí solo posibilita todas las facilidades para su buen entendimiento porque trae consigo toda la información y el corpus lingüístico que materializa la temática; por lo tanto, exige al lector habilidades de análisis, síntesis, vocabulario y conocimientos de los recursos lingüísticos para que no desaproveche la riqueza de construcción, significado e información del texto. No todos los lectores presentan por igual las mismas destrezas lectoras, unos son más lentos, otros rápidos; algunos manejan técnicas y procedimientos, otros no, etc. Viendo esta problemática es necesario estudiar las diferencias individuales que presentan los estudiantes en su vida académica y por ende en la lectura. El entendimiento de este fenómeno podría ser la clave para enfrentarnos en forma adecuada en el tratamiento acerca de la comprensión de lectura en las aulas universitarias. 
La comprensión lectora como parte de la competencia lingüística involucra procesos cognitivos complejos que ameriten su estudio. Como se ha manifestado, cada individuo presenta peculiaridades en la ejecución de sus actividades diversas y más aún si estos están relacionados con la lectura. Cada individuo posee características distintas al momento de leer y comprender un texto. Esto se conoce como las diferencias individuales y en nuestro caso serían los estilos de aprendizaje. Por cierto, existen estilos que favorecen mejor la comprensión lectora que otros. Estos estilos se presentan en parejas (teórico/reflexivo, activo/pragmático, etc.) que funcionan en forma adecuada con la persona adecuada. Esto no significa que un estilo, de ahí nace la controversia, es mejor que otro. Sin embargo, el respeto a las formas de lectura, sus procedimientos, habilidades, entre otros, podría ser la clave para mejorar la comprensión de lectura y por ende el rendimiento académico. Esto no significa que se descuide las otras variables que inciden en el resultado lector.

Es de suponer que exista una interacción entre los estilos de aprendizaje y la comprensión de lectura, las evidencias científicas que respaldan esta postura son las siguientes: Manzano e Hidalgo (2009), Briceño et al. (2011), Barboza y Galván (2013), Marcelo y Sedano (2013), Moreno (2014), Jara et al. (2015), Rodríguez et al. (2015), Vásquez y Llontop (2015), Zárate (2015), Tangoa e Hidalgo (2016), Loayza (2017) y Mendoza (2018).

\section{Materiales y métodos}

Investigación no experimental, transeccional con diseño descriptivo comparativo, cuya población objetiva estuvo constituida por los estudiantes universitarios de Huancayo. La muestra seleccionada intencionalmente estuvo conformada por 300 estudiantes universitarios: 181 de la Universidad Nacional del Centro del Perú y 119 de la Universidad Continental; 107 del género masculino y 193 del femenino cuyas edades oscilaban entre 16 a 48 años.

La técnica para recolectar la información de la variable X: fue la prueba pedagógica de comprensión de textos validada y confiabilizada por Palacios (2005) y para la variable Y: el cuestionario de estilos de aprendizaje de Honey - Alonso (CHAE).

Para el análisis estadístico se hizo uso de la estadística descriptiva, a través de las frecuencias y de la estadística inferencial, $\mathrm{Ch}^{2}$.

\section{Resultados}

\section{Comprensión de textos}

La comprensión de textos predominante en los estudiantes universitario de Huancayo.

Tabla 1

Niveles de comprensión de textos en la muestra total

\begin{tabular}{lc|c}
\hline \multirow{2}{*}{ Niveles de comprensión de textos } & \multicolumn{2}{c}{ Muestra total } \\
\cline { 2 - 3 } & $\mathbf{N i}$ & $\%$ \\
\hline Alto & 0 & 0 \\
Medio & 85 & 28 \\
Bajo & 215 & 72 \\
\hline Total & 300 & 100 \\
\hline
\end{tabular}


En la tabla 1 se exhiben los resultados obtenidos de los niveles de comprensión de textos, mediante la prueba pedagógica, de los estudiantes universitarios de Huancayo.

Se observa que predomina el nivel bajo (72\%), seguido del nivel medio (28\%) y alto (o\%).

\section{Comprensión de textos en función de las variables de control}

Género

Tabla 2

Niveles de comprensión de textos según sexo

\begin{tabular}{lcccc}
\hline & \multicolumn{2}{c}{ Masculino } & \multicolumn{2}{c}{ Femenino } \\
\hline Niveles (CT) & (fi) & \% & (fi) & \% \\
\hline Alto & 0 & 0 & 0 & 0 \\
Medio & 29 & 27 & 56 & 29 \\
Bajo & 78 & 73 & 137 & 71 \\
\hline Total & $\mathbf{1 0 7}$ & $\mathbf{1 0 0}$ & $\mathbf{1 9 3}$ & $\mathbf{1 0 0}$ \\
\hline
\end{tabular}

En la tabla 2 se exhiben los resultados obtenidos según sexo.

Se observa que en el sexo masculino predomina el nivel bajo (73\%), seguido del nivel medio (29\%) y alto (o\%). Mientras que en el sexo femenino predomina el nivel bajo ( $71 \%)$, seguido del medio (29\%) y el alto (0\%).

\section{Edad}

Atendiendo a la edad, en vista de que había estudiantes de 16 años hasta los 48 , se estableció dos grupos: menores de 20 años y mayores de 20 años.

Tabla 3

Niveles de comprensión de textos según edad

\begin{tabular}{lcccc}
\hline & \multicolumn{2}{c}{ Menores de 20 años } & \multicolumn{2}{c}{ Mayores de 20 años } \\
\hline Nivel (CT) & (fi) & $\%$ & (fi) & \% \\
\hline Alto & 0 & 0 & 0 & 0 \\
Medio & 61 & 28 & 24 & 29 \\
Bajo & 157 & 72 & 58 & 71 \\
\hline Total & $\mathbf{2 1 8}$ & $\mathbf{1 0 0}$ & $\mathbf{8 2}$ & $\mathbf{1 0 0}$ \\
\hline
\end{tabular}

En la tabla 3 se exhiben los resultados obtenidos según la edad. Se observa que en los menores de 20 años predomina el nivel bajo (72\%), seguido del nivel medio (28\%) y alto (0\%). Mientras que en los mayores de 20 años predomina el nivel bajo (71\%), seguido del medio (29\%) y el alto (o\%). 


\section{Condición económica}

Según este variable de control, este grupo se clasifica en condición económica baja, media y alta.

Tabla 4

Niveles de comprensión de textos según condición económica

\begin{tabular}{lcccccc}
\hline & & Baja & \multicolumn{2}{c}{ Media } & \multicolumn{2}{c}{ Alta } \\
\hline Nivel (CT) & (fi) & $\%$ & (fi) & $\%$ & (fi) & $\%$ \\
\cline { 3 - 7 } Alto & 0 & 0 & 0 & 0 & 0 & 0 \\
Medio & 19 & 40 & 64 & 26 & 2 & 22 \\
Bajo & 28 & 60 & 180 & 74 & 7 & 78 \\
\hline Total & 47 & 100 & 244 & 100 & 9 & 100 \\
\hline
\end{tabular}

En la tabla 4 se exhiben los resultados obtenidos según condición económica. Se observa que en la clase baja predomina el nivel bajo (58.33\%), seguido del nivel medio (41.67\%) y alto (०\%). Mientras que en la clase media predomina el nivel bajo (67.38\%), seguido del medio (32.62\%) y el alto (0\%). Asimismo, en la clase alta predomina el nivel bajo (75\%), seguido del medio (25\%) y el alto (o\%).

\section{Condición familiar}

Según esta variable de control, esta se clasifica en estudiantes que viven con papá y mamá, con papá, con mamá, con algún familiar y aquellos que viven solos.

Tabla 5

Niveles de comprensión de textos según condición familiar

\begin{tabular}{|c|c|c|c|c|c|c|c|c|c|c|}
\hline \multirow[b]{2}{*}{ Nivel (CT) } & \multicolumn{2}{|c|}{ Papá y mamá } & \multicolumn{2}{|c|}{ Papá } & \multicolumn{2}{|c|}{ Mamá } & \multicolumn{2}{|c|}{ Familiar } & \multicolumn{2}{|c|}{ Solo } \\
\hline & (fi) & $\%$ & (fi) & $\%$ & (fi) & $\%$ & (fi) & $\%$ & (fi) & $\%$ \\
\hline Alto & 0 & o & 0 & 0.00 & 0 & 0 & 0 & o & o & o \\
\hline Medio & 43 & 27 & 0 & 0.00 & 17 & 30 & 10 & 30 & 15 & 31 \\
\hline Bajo & 114 & 73 & 4 & 100 & 40 & 70 & 23 & 70 & 34 & 69 \\
\hline Total & 157 & 100 & 4 & 100 & 57 & 100 & 33 & 100 & 49 & 100 \\
\hline
\end{tabular}

En la tabla 5 se exhiben los resultados obtenidos según condición familiar. Se observa que en los estudiantes que viven con papá y mamá predomina el nivel bajo (73\%), seguido del nivel medio (27\%) y alto (0\%). Mientras que en los estudiantes que viven con papá predomina el nivel bajo (100\%), seguido del medio (0\%) y el alto (0\%). Asimismo, en los estudiantes que viven con mamá predomina el nivel bajo (70\%), seguido del medio (30\%) y el alto (o\%). Por otro lado, los estudiantes que viven con un familiar predominan el nivel bajo (70\%), seguido 
del medio (30\%) y el alto (०\%). Por último, los estudiantes que viven solo, predominan el nivel bajo (69\%), seguido del medio (31\%) y el alto (0\%).

\section{Estilos de aprendizaje}

Esta variable de control se clasifica en estudiantes cuyos padres no tienen estudios, con estudios primarios y secundarios.

\section{Tabla 6}

Niveles de comprensión de textos según estilos de aprendizaje

\begin{tabular}{|c|c|c|c|c|c|c|c|c|}
\hline \multirow[b]{2}{*}{ Nivel (CT) } & \multicolumn{2}{|c|}{ Activo } & \multicolumn{2}{|c|}{ Reflexivo } & \multicolumn{2}{|c|}{ Teórico } & \multicolumn{2}{|c|}{ Pragmático } \\
\hline & (fi) & $\%$ & (fi) & $\%$ & (fi) & $\%$ & (fi) & $\%$ \\
\hline Alto & o & o & o & o & o & o & 0 & o \\
\hline Medio & 25 & 32 & 27 & 28 & 14 & 21 & 19 & 33 \\
\hline Bajo & 53 & 68 & 70 & 72 & 53 & 79 & 39 & 67 \\
\hline Total & 78 & 100 & 97 & 100 & 67 & 100 & 58 & 100 \\
\hline
\end{tabular}

En la tabla 6 se exhiben los resultados obtenidos según el estilo de aprendizaje. Se observa que en los estudiantes con el estilo activo predomina el nivel bajo (68\%), seguido del nivel medio (32\%) y alto (o\%). Mientras que en los estudiantes con el estilo reflexivo predomina el nivel bajo (72\%), seguido del medio (28\%) y el alto (o\%). Asimismo, en los estudiantes con el estilo teórico predominan el nivel bajo (79\%), seguido del medio (19\%) y el alto (o\%). Por último, en los estudiantes con el estilo pragmático predominan el nivel bajo (67\%), seguido del medio (33\%) y el alto (o\%).

\section{Comparación de la comprensión de texto}

\section{Género}

Para realizar la comparación, se recurrió al estadístico Chi cuadrada $\left(\mathrm{X}^{2}\right)$.

\section{Hipótesis de trabajo}

Ho: No existen diferencias significativas entre los puntajes de la comprensión de textos de los estudiantes universitarios de la muestra de estudio según la categoría género al o,05 de probabilidad de error.

Ha: No existen diferencias significativas entre los puntajes de la comprensión de textos de los estudiantes universitarios de la muestra de estudio según la categoría género al o,05 de probabilidad de error. 


\section{Cálculo del estadígrafo de prueba}

Tabla 7

Puntaje Chi cuadrada de comprensión de textos según género

\begin{tabular}{lcccc}
\hline \multirow{2}{*}{$\begin{array}{l}\text { Niveles de comprensión de } \\
\text { textos }\end{array}$} & \multicolumn{2}{c}{ Masculino } & \multicolumn{2}{c}{ Femenino } \\
\cline { 2 - 5 } & fo & fe & fo & fe \\
\hline Alto & 0 & 0 & $\circ$ & 0 \\
Medio & 29 & 30.32 & 56 & 54.68 \\
Bajo & 78 & 76.68 & 137 & 138.32 \\
\hline Total & $\mathbf{1 0 7}$ & & $\mathbf{1 9 3}$ & \\
\hline
\end{tabular}

Procesando tenemos:

\begin{tabular}{|c|c|c|c|c|}
\hline fo & fe & fo-fe & $(\text { fo-fe })^{2}$ & $\frac{(\text { fo-fe })^{2}}{f e}$ \\
\hline o & 0 & o & o & o \\
\hline 29 & 30.32 & -1.32 & 1.7424 & 0.05746702 \\
\hline 78 & 76.68 & 1.32 & 1.7424 & 0.022723 \\
\hline 0 & o & $\mathrm{o}$ & o & o \\
\hline 56 & 54.68 & 1.32 & 1.7424 & 0.0318654 \\
\hline 137 & 138.32 & -1.32 & 1.7424 & 0.01259688 \\
\hline
\end{tabular}

$X^{2} \mathrm{C}=0.1246523$

\section{Decisión}

Como $X^{2} c$ con datos muestrales es < que la $X^{2} t$ (0.1246523 < que 5.992) se acepta la Ho y como consecuencia se rechaza la $\mathrm{Ha}$; es decir no existe diferencias significativas entre los puntajes frecuenciales de comprensión de textos de los estudiantes universitarios según la categoría género al 0.05 de probabilidad de error.

\section{Edad}

Para realizar la comparación, se recurrió al estadístico Chi cuadrada $\left(\mathrm{X}^{2}\right)$.

\section{Hipótesis de trabajo}

Ho: No existen diferencias significativas entre los puntajes de la comprensión de textos de los estudiantes universitarios de la muestra de estudio según la categoría edad al o,05 de probabilidad de error.

Ha: No existen diferencias significativas entre los puntajes de la comprensión de textos de los estudiantes universitarios de la muestra de estudio según la categoría edad al o,05 de probabilidad de error. 


\section{Cálculo del estadígrafo de prueba}

Tabla 8

Puntaje Chi cuadrada de comprensión de textos según edad

\begin{tabular}{lcccc}
\hline Niveles de comprensión de & \multicolumn{2}{c}{ < de 20 años } & \multicolumn{2}{c}{ > de 20 años } \\
\cline { 2 - 5 } textos & fo & fe & fo & fe \\
\hline Alto & 0 & 0 & $\circ$ & 0 \\
Medio & 61 & 61.77 & 24 & 23.23 \\
Bajo & 157 & 156.23 & 58 & 58.77 \\
\hline Total & $\mathbf{2 1 8}$ & & $\mathbf{8 2}$ & \\
\hline
\end{tabular}

Procesando tenemos:

\begin{tabular}{|c|c|c|c|c|}
\hline fo & fe & fo-fe & $(\text { fo-fe })^{2}$ & $\frac{(\text { fo-fe })^{2}}{f e}$ \\
\hline 0 & o & 0 & o & o \\
\hline 61 & 61.77 & -0.77 & 0.5929 & 0.00959851 \\
\hline 157 & 156.23 & 0.77 & 0.5929 & 0.00379505 \\
\hline 0 & 0 & o & o & o \\
\hline 24 & 23.23 & 0.77 & 0.5929 & 0.02552303 \\
\hline 58 & 58.77 & -0.77 & 0.5929 & 0.01008848 \\
\hline
\end{tabular}

$X^{2} \mathrm{C}=0.04900507$

Decisión: Como $X^{2} c$ con datos muestrales es < que la $X^{2} t$ (0.04900507 < que 5.992) se acepta la Ho y como consecuencia se rechaza la $\mathrm{Ha}$; es decir no existe diferencias significativas entre los puntajes frecuenciales de comprensión de textos de los estudiantes universitarios según la categoría edad al 0.05 de probabilidad de error.

\section{Condición económica}

Para realizar la comparación, se recurrió al estadístico Chi cuadrada $\left(\mathrm{X}^{2}\right)$.

\section{Hipótesis de trabajo}

Ho: No existen diferencias significativas entre los puntajes de la comprensión de textos de los estudiantes universitarios de la muestra de estudio según la categoría condición económica al 0,05 de probabilidad de error.

Ha: No existen diferencias significativas entre los puntajes de la comprensión de textos de los estudiantes universitarios de la muestra de estudio según la categoría condición económica al 0,05 de probabilidad de error. 


\section{Cálculo del estadígrafo de prueba}

Tabla 9

Puntaje Chi cuadrada de comprensión de textos según condición económica

\begin{tabular}{lcccccc}
\hline \multirow{2}{*}{$\begin{array}{l}\text { Niveles de comprensión de } \\
\text { textos }\end{array}$} & \multicolumn{2}{c}{ Baja } & \multicolumn{2}{c}{ Media } & \multicolumn{2}{c}{ Alta } \\
\cline { 2 - 8 } & fe & fo & fe & fo & fe & fo \\
\hline Alto & $\circ$ & $\circ$ & $\circ$ & $\circ$ & $\circ$ & 0 \\
Medio & 19 & 10.44 & 64 & 69.13 & 2 & 2.55 \\
Bajo & 28 & 36.56 & 180 & 174.87 & 7 & 6.45 \\
\hline Total & $\mathbf{4 7}$ & & $\mathbf{2 4 4}$ & & $\mathbf{9}$ & \\
\hline
\end{tabular}

Procesando tenemos:

\begin{tabular}{|c|c|c|c|c|}
\hline fo & fe & fo-fe & ${\text { (fo-fe })^{2}}^{\mathbf{( f o - f e})^{\mathbf{2}}}$ & fe \\
\hline 0 & 0 & 0 & 0 & 0 \\
\hline 19 & 10.44 & 8.56 & 73.2736 & 7.01854406 \\
\hline 28 & 36.56 & -8.56 & 73.2736 & 2.00420131 \\
\hline 0 & 0 & 0 & 0 & 0 \\
\hline 64 & 69.13 & -5.13 & 26.3169 & 0.38068711 \\
\hline 180 & 174.87 & 5.13 & 26.3169 & 0.15049408 \\
\hline 0 & 0.00 & 0 & 0 & 0 \\
\hline 2 & 2.55 & -0.55 & 0.3025 & 0.11862745 \\
\hline 7 & 6.45 & 0.55 & 0.3025 & 0.04689922 \\
\hline
\end{tabular}

$X^{2} \mathrm{C}=9.71945324$

Decisión: Como $X^{2} c$ con datos muestrales es < que la $X^{2} t(9.71945324$ < que 9.49) se acepta la Ha y como consecuencia se rechaza la Ho; es decir existe diferencias significativas entre los puntajes frecuenciales de comprensión de textos de los estudiantes universitarios según la categoría condición económica al 0.05 de probabilidad de error.

\section{Condición familiar}

Para realizar la comparación, se recurrió al estadístico Chi cuadrada $\left(\mathrm{X}^{2}\right)$.

\section{Hipótesis de trabajo}

Ho: No existen diferencias significativas entre los puntajes de la comprensión de textos de los estudiantes universitarios de la muestra de estudio según la categoría condición familiar al o,05 de probabilidad de error.

Ha: No existen diferencias significativas entre los puntajes de la comprensión de textos de los estudiantes universitarios de la muestra de estudio según la categoría condición familiar al o,05 de probabilidad de error. 


\section{Cálculo del estadígrafo de prueba}

\section{Tabla 10}

Puntajes Chi cuadrada de la comprensión de textos según condición familiar

\begin{tabular}{|c|c|c|c|c|c|c|c|c|c|c|}
\hline \multirow{2}{*}{$\begin{array}{l}\text { Niveles de } \\
\text { comprensión } \\
\text { de texto }\end{array}$} & \multicolumn{2}{|c|}{ Papá y mamá } & \multicolumn{2}{|c|}{ Papá } & \multicolumn{2}{|c|}{ Mamá } & \multicolumn{2}{|c|}{ Familiar } & \multicolumn{2}{|c|}{ Solo } \\
\hline & fo & fe & fo & fe & fo & fe & fo & fe & fo & fe \\
\hline Alto & 0 & o & o & o & 0 & o & o & o & 0 & o \\
\hline Medio & 43 & 46.82 & 0 & 1.13 & 17 & 16.15 & 10 & 9.35 & 15 & 28.33 \\
\hline Bajo & 114 & 110.18 & 4 & 2.87 & 40 & 40.85 & 23 & 23.65 & 60 & 71.67 \\
\hline Total & 157 & & 4 & & 57 & & 33 & & 100 & \\
\hline
\end{tabular}

Procesando tenemos:

\begin{tabular}{|c|c|c|c|c|}
\hline fo & fe & fo-fe & (fo-fe) 2 & $\frac{(f o-f e)^{2}}{f e}$ \\
\hline 0 & 0 & o & 0 & o \\
\hline 43 & 46.82 & -3.82 & 14.5924 & 0.31167 \\
\hline 114 & 110.18 & 3.82 & 14.5924 & 0.132441 \\
\hline 0 & 0 & 0 & 0 & 0 \\
\hline 0 & 1.13 & -1.13 & 1.2769 & 1.13 \\
\hline 4 & 2.87 & 1.13 & 1.2769 & 0.444913 \\
\hline 0 & 0.00 & 0 & 0 & 0 \\
\hline 17 & 16.15 & 0.85 & 0.7225 & 0.044737 \\
\hline 40 & 40.85 & -0.85 & 0.7225 & 0.017687 \\
\hline 0 & 0.00 & 0 & 0 & 0 \\
\hline 10 & 9.35 & 0.65 & 0.4225 & 0.045187 \\
\hline 23 & 23.65 & -0.65 & 0.4225 & 0.017865 \\
\hline 0 & 0.00 & 0 & 0 & 0 \\
\hline 31 & 28.33 & 2.67 & 7.1289 & 0.251638 \\
\hline 69 & 71.67 & -2.67 & 7.1289 & 0.099468 \\
\hline
\end{tabular}

$X^{2} \mathrm{C}=\mathbf{2 . 4 9 5 6 0 6}$

Decisión: Como $X^{2} c$ con datos muestrales es < que la X2t $(2.495606$ < que 15.51) se acepta la Ho y como consecuencia se rechaza la $\mathrm{Ha}$; es decir no existe diferencias significativas entre los puntajes frecuenciales de comprensión de textos de los estudiantes universitarios según la categoría condición familiar al 0.05 de probabilidad de error.

\section{Estilos de aprendizaje}

Para realizar la comparación, se recurrió al estadístico Chi cuadrada $\left(\mathrm{X}^{2}\right)$. 


\section{Hipótesis de trabajo}

Ho: No existen diferencias significativas entre los puntajes de la comprensión de textos de los estudiantes universitarios de la muestra de estudio según la categoría estilos de aprendizaje al o,05 de probabilidad de error.

Ha: No existen diferencias significativas entre los puntajes de la comprensión de textos de los estudiantes universitarios de la muestra de estudio según la categoría estilos de aprendizaje al o,05 de probabilidad de error.

\section{Cálculo del estadígrafo de prueba}

Tabla 11

Puntaje Chi cuadrada de la comprensión de textos según estilos de aprendizaje

\begin{tabular}{lccccccccc}
\hline $\begin{array}{l}\text { Niveles } \\
\text { comprensión } \\
\text { textos }\end{array}$ & $\begin{array}{l}\text { de } \\
\text { de }\end{array}$ & \multicolumn{2}{c}{ Activo } & \multicolumn{2}{c}{ Reflexivo } & \multicolumn{2}{c}{ Teórico } & \multicolumn{2}{c}{ Pragmático } \\
\cline { 2 - 10 } & fo & fe & fo & fe & fo & fe & fo & fe \\
\hline Alto & 0 & $\circ$ & $\circ$ & $\circ$ & 0 & 0 & 0 & 0 \\
Medio & 25 & 22.10 & 27 & 27.48 & 14 & 18.98 & 19 & 16.43 \\
Bajo & 53 & 55.90 & 70 & 69.52 & 53 & 48.02 & 39 & 41.57 \\
\hline Total & $\mathbf{7 8}$ & & $\mathbf{9 7}$ & & $\mathbf{6 7}$ & & $\mathbf{5 8}$ & \\
\hline
\end{tabular}

Procesando tenemos:

\begin{tabular}{|c|c|c|c|c|}
\hline fo & fe & fo-fe & (fo-fe) ${ }^{2}$ & $\begin{array}{c}\text { (fo-fe) }^{2} \\
\text { fe }\end{array}$ \\
\hline 0 & 0 & 0 & 0 & 0 \\
\hline 25 & 22.10 & 2.9 & 8.41 & 0.380543 \\
\hline 53 & 55.90 & -2.9 & 8.41 & 0.150447 \\
\hline 0 & 0 & 0 & 0 & 0 \\
\hline 27 & 27.48 & -0.48 & 0.2304 & 0.008384 \\
\hline 70 & 69.52 & 0.48 & 0.2304 & 0.003314 \\
\hline 0 & 0.00 & 0 & 0 & 0 \\
\hline 14 & 18.98 & -4.98 & 24.8004 & 1.30666 \\
\hline 53 & 48.02 & 4.98 & 24.8004 & 0.51646 \\
\hline 0 & 0 & 0 & 0 & 0 \\
\hline 19 & 16.43 & 2.57 & 6.6049 & 0.402002 \\
\hline 39 & 41.57 & -2.57 & 6.6049 & 0.158886 \\
\hline
\end{tabular}

$X^{2} \mathrm{C}=2.926697$

Decisión: Como $X^{2} c$ con datos muestrales es < que la X²t (2.926697 < que 12.59) se acepta la Ho y como consecuencia se rechaza la $\mathrm{Ha}$; es decir no existe diferencias significativas entre los puntajes frecuenciales de comprensión de textos de los estudiantes universitarios según la categoría estilos de aprendizaje al 0.05 de probabilidad de error. 


\section{Discusión}

El rendimiento de la comprensión lectora en estos últimos años, sobre todo en nuestro medio, no ha variado. Los resultados evidencian un bajo nivel de comprensión lectora ya en primaria, secundaria y universidad. Estos datos corroboran la evaluación ECE y PISA. Sin duda, la crisis educativa ha llegado a niveles extremos. La educación peruana, como tal ha pasado a un segundo plano, más importante es la corrupción, el dinero y el tráfico de influencias.

Esta investigación evidencia el bajo nivel de lectura en los estudiantes universitarios de Huancayo, este dato es semejante a los hallados por Inga (2017), Palacios (2018) y Palacios (2019) en el nivel universitario; Quinto (2014), Castro (2014), Vera (2014) y Capcha (2015) en el nivel secundario.

Con respecto a las variables de control género, sexo, condición económica, condición familiar en relación a la comprensión lectora los resultados presentan un bajo nivel de lectura, este dato se corrobora con las investigaciones de Palacios (2018) y Palacios (2019) en el nivel universitario; Quinto (2014), Castro (2014), Vera (2014) en el nivel secundario.

Los estilos de aprendizaje son las diversas actitudes y capacidades que pone en práctica un estudiante al momento de la obtención, procesamiento y recreación de la información. En esta investigación los estudiantes universitarios de Huancayo se caracterizan por presentar un estilo reflexivo seguido del activo.

No se encontró una relación objetiva entre la comprensión de textos y los estilos de aprendizaje, este resultado es similar a lo hallado por Contijoch y Popoca (1999), Zavala (2008), Wong y Matalinares (2011), Padilla (2016). En nuestro caso, no existe diferencias significativas entre la comprensión de textos y los estilos de aprendizaje. Este aspecto es muy importante porque la comprensión lectora es la sumatoria de la intervención de diversas variables y el estilo de aprendizaje es uno de ellos. Su intervención quizá no sea significativa, pero es necesario determinarlo, cultivarlo y perfeccionarlo porque es la clave para el mejoramiento, no solo de la comprensión de lectura, sino del rendimiento académico.

\section{Conclusiones}

La comprensión de textos que presentan los estudiantes universitarios de Huancayo es de nivel bajo. Del 100\% (300) de estudiantes el 72\% (215) presentan un nivel bajo; el $28 \%$ (85), un nivel medio; $y$, ningún estudiante se encuentra en el alto.

El estilo de aprendizaje que predomina en lo estudiantes universitarios de Huancayo es el reflexivo seguido del activo.

Según la variable género, los estudiantes universitarios de Huancayo se encuentran en el nivel bajo de comprensión de textos: sexo masculino $(\mathrm{Ma}=10.71)$ y sexo femenino $(\mathrm{Ma}=10.48)$.

Según la variable edad, los estudiantes universitarios de Huancayo se encuentran en el nivel bajo de comprensión de textos: estudiantes menores de 20 años $(\mathrm{Ma}=10.63)$ y estudiantes mayores de 20 años $(\mathrm{Ma}=10.38)$. 
Según la variable condición económica, los estudiantes universitarios de Huancayo se encuentran en el nivel bajo de comprensión de textos: estudiantes de la condición económica alta $(\mathrm{Ma}=9.11)$, estudiantes de la condición económica media $(\mathrm{Ma}=11.3)$ y estudiantes de la condición económica baja $(\mathrm{Ma}=10.48)$.

Según la variable condición familiar, los estudiantes universitarios de Huancayo se encuentran en el nivel bajo de comprensión de textos: estudiantes que viven con papá y mamá ( $\mathrm{Ma}=$ 10.54), estudiantes que viven con papá ( $M a=9.5)$, estudiantes que viven con mamá $(M a=10.7)$, estudiantes que viven con algún familiar $(M a=10.12)$ y estudiantes que viven solos $(M a=10.88)$.

Según la variable estilos de aprendizaje, los estudiantes universitarios de Huancayo se encuentran en el nivel bajo de comprensión de textos: estudiantes con estilo de aprendizaje activo $(\mathrm{Ma}=10.95)$, estudiantes con estilo de aprendizaje reflexivo $(\mathrm{Ma}=10.6)$, estudiantes con estilo de aprendizaje teórico $(\mathrm{Ma}=9.96)$ y estudiantes con estilo de aprendizaje pragmático $(\mathrm{Ma}=10.71)$.

No existe diferencias significativas entre la comprensión de texto y los estilos de aprendizaje.

No existe diferencias significativas entre la comprensión de textos y la variable de control género $X_{2 C}=0.1246523$ es menor que la X2t $=5.99$ a un nivel de confianza del $95 \%$ de acierto 0 $5 \%$ de margen de error.

No existe diferencias significativas entre la comprensión de textos y la variable de control edad X $2 \mathrm{C}=0.0490057$ es menor que la X2t $=5.99$ a un nivel de confianza del $95 \%$ de acierto 0 $5 \%$ de margen de error.

Existe diferencias significativas entre la comprensión de textos y la variable de control condición económica X2C $=9.71945324$ es mayor que la X2t $=9.49$ a un nivel de confianza del $95 \%$ de acierto o $5 \%$ de margen de error.

No existe diferencias significativas entre la comprensión de textos y la variable de control condición familiar X2C $=2.495606$ es menor que la X2t $=15.51$ a un nivel de confianza del $95 \%$ de acierto $05 \%$ de margen de error.

No existe diferencias significativas entre la comprensión de textos y la variable de control estilos de aprendizaje $\mathrm{X}_{2 \mathrm{C}}=\mathbf{2 . 9 2 6 6 9 7}$ es menor que la $\mathrm{X}_{2 \mathrm{t}}=12.59$ a un nivel de confianza del $95 \%$ de acierto $05 \%$ de margen de error. 
Barboza, Y. E. y Galván, M. (2013). Estilos de aprendizaje y estilos de enseñanza: una relación necesaria en la comprensión lectora en inglés. [Tesis para obtener el título de Magister en Educación]. Facultad de Educación. Universidad de Antioquía.

Briceño, L., Rojas, F., y Peinado, S. (2011). Influencia de los mapas conceptuales y los estilos de aprendizaje en la comprensión de la lectura. Revista Estilos de Aprendizaje, $n^{\circ} 8$, Vol. 4, octubre de 2011.

Capcha, A. V. (2015). La comprensión de textos expositivos de los estudiantes del sexto grado de educación primaria de las instituciones educativas estatales del distrito metropolitano de EI Tambo. [Tesis para optar el grado de Magister, mención Enseñanza estratégica]. Facultad de Educación. Universidad Nacional del Centro del Perú.

Castro, R. T. (2014). La comprensión de textos expositivos de los estudiantes del cuarto grado de educación secundaria de las instituciones educativas estatales de la zona sur del distrito de Huancayo. [Tesis para optar el grado de maestría, mención Lectura y escritura]. Facultad de Educación Universidad Nacional del Centro del Perú.

Contijoch, M., y Popoca, L. (1999). Relación entre estilo de aprendizaje independiente/dependiente de campo y comprensión de lectura. Estudios de Lingüística Aplica, núm. 29, 1999.

Inga, R. (2017). Comprensión de texto y clima social familiar en estudiantes de Ingeniería y Ciencias Humanas - UNCP. [Tesis para optar el Grado Académico de Maestro en Educación, mención Educación Superior]. Facultad de Educación. Universidad Nacional del Centro del Perú.

Jara, M., Pumahuilca, S., y Valdivia, C. P. (2015). Estilos de aprendizaje y la comprensión de lectura de los estudiantes de 5 to grado de Educación Primaria, de la Institución Educativa $N^{\circ} 0137$ Miguel Grau Seminario del distrito de San Juan de Lurigancho, Ugel: 05. [Tesis para optar el Título Profesional de Licenciado en Educación]. Facultad de Pedagogía y Cultura Física. Universidad Nacional de Educación Enrique Guzmán y Valle.

Loayza, M. (2017). Estilos de aprendizaje y comprensión lectora en estudiantes de 4to. grado del colegio Santa Rosa, Huacho - 2017. [Tesis para optar el grado académico de Maestra en Psicología Educativa]. Universidad César Vallejo.

Marcelo, M. Z., y Sedano, M. M. (2013). Relación entre estilos de aprendizaje Vak y comprensión lectora de los estudiantes del Tercer Grado de Secundaria de la I.E. Miguel Grau Seminario de San Juan de Lurigancho. [Tesis para optar el grado de Magister en Psicología Educativa]. Universidad César Vallejo.

Manzano, M., y Hidalgo, E. (2009). Estilos de aprendizaje, estrategias de lectura y su relación con el rendimiento académico de la lengua extranjera. Educación XXI. 12, 2009, pp. 123-150. Facultad de Educación, UNED.

Mendoza, G. E. (2018). Los estilos de aprendizaje y su relación con la comprensión de lectura en el idioma inglés en los estudiantes del tercer grado de secundaria de la I.E. $N^{\circ} 1199$ Mariscal Ramón Castilla de Chaclacayo, 2018. [Tesis para optar el título profesional de Licenciada en Educación, especialidad Lingüística e Inglés]. Facultad de Ciencias Humanas y Educación. Universidad Peruana Unión.

Moreno, L. N. (2014). Los estilos de aprendizaje y su relación con la comprensión lectora de los estudiantes del sexto grado de educación primaria de la I.E. Simón Bolívar de Independencia - Huaraz - Ancash. [Tesis para optar el título de Licenciada en Educación, especialidad: Primaria y Educación Bilingüe Intercultural]. Facultad de Ciencias Sociales, Educación y de la Comunicación. Universidad Nacional Santiago Antúnez de Mayolo.

Padilla, A. C. (2016). Estilos de aprendizaje y comprensión lectora en estudiantes de sexto grado del distrito de Lima. [Tesis para obtener el título profesional de Licenciatura en Educación Primaria]. Facultad de Educación e Idiomas. Universidad César Vallejo.

Palacios, M. A. (2005). El enfoque comunicativo en la comprensión de textos expositivos de los alumnos universitarios de Huancayo. [Tesis para optar el Grado Académico de Magister en Educación, mención Didáctica Universitaria]. Facultad de Educación. Universidad Nacional del Centro del Perú. 
Palacios, M. A. (2018). Comprensión de textos e inteligencia emocional en los estudiantes universitarios de Huancayo. Instituto especializado de Investigación de la Facultad de Educación. Universidad Nacional del Centro del Perú.

Palacios, M. A. (2019). Autoestima y comprensión de texto en los estudiantes universitarios de la UNCP. Instituto especializado de Investigación de la Facultad de Educación. Universidad Nacional del Centro del Perú.

Quinto, E. P. (2014). Aptitudes mentales primarias y la comprensión de textos de los estudiantes de quinto grado de educación secundaria de las instituciones educativas estatales del distrito de Satipo. [Tesis para optar el grado de Magister, mención Lectura y escritura]. Facultad de Educación. Universidad Nacional del Centro del Perú.

Rodríguez, R. C., Zárate, J. F., \& Rodríguez, A. (2015). Los estilos de aprendizaje, hábitos de lectura y la comprensión lectora a través del uso del hipertexto en estudiantes de bachillerato. Revista de estilos de aprendizaje. Vol. $9 N^{\circ} 18$

Tangoa, N., e Hidalgo, G. (2016). Estilos de aprendizaje y comprensión lectora de los estudiantes del quinto grado de educación primaria de la institución educativa $N^{\circ} 62313$ de Palmichi del pueble de Shawi Cahuapanas - Loreto 2015. [Tesis para optar el título profesional de Licenciado en Educación Primaria Bilingüe]. Facultad de Educación Intercultural y Humanidades. Universidad Nacional Intercultural de la Amazonía.

Vásquez, G. E., y Llontop, J. C. (2015). Los estilos de aprendizaje y su relación con la comprensión lectora en los estudiantes del tercer grado de secundario del Colegio Experimental de Aplicación de la Universidad Nacional de Educación "Enrique Guzmán y Valle. [Tesis para optar el título profesional de Licenciado en Educación Especialidad: Inglés - Francés]. Facultad de Ciencias Sociales y Humanidades, Escuela Profesional de Lenguas Extranjeras y Nativas. Universidad Nacional de Educación Enrique Guzmán y Valle.

Vera, R. C. (2014). La comprensión de textos expositivos de los estudiantes del Quinto Grado de Educación Secundaria de las Instituciones Educativas Estatales de El Tambo. [Tesis para optar el Grado Académico de Magister en Educación, mención Lectura y Escritura]. Facultad de Educación. Universidad Nacional del Centro del Perú.

Wong, F., y Matalinares, M. (2011). Estrategias de metacomprensión lectora y estilos de aprendizaje en estudiantes universitarios. Revista IIPSI. Facultad de Psicología. UNMSM. Vol. $14-\mathrm{N}^{\circ} 1-2011$, pp. $235-260$.

Zárate, G. M. (2015). Los estilos de aprendizaje y su relación con la comprensión lectora en los estudiantes del nivel secundario de las instituciones educativas de Ate - UGEL $N^{\circ} 06$ Ate Vitarte. [Tesis para optar el grado académico de Magister en Ciencias de la Educación, mención Problemas de aprendizaje]. Universidad Nacional de Educación Enrique Guzmán y Valle.

Zavala, H. A. (2008). Relación entre el estilo de aprendizaje y el nivel de comprensión lectora en estudiantes de 5to. de secundaria de colegios estatales y particulares de Lima Metropolitana. [Tesis para optar el grado académico de Magister en Psicología con mención Psicología Educativa]. Facultad de Psicología. Universidad Nacional Mayor de San Marcos. 